\title{
Fuzzy Logic Based Decision Making Algorithm to Optimize the Handover Performance in HetNets
}

\author{
A. Prithiviraj ${ }^{1}$, K. Krishnamoorthy ${ }^{2}$, B. Vinothini ${ }^{1}$ \\ ${ }^{1}$ Computer Science and Engineering, Sona College of Technology, Salem, India \\ ${ }^{2}$ Computer Science and Engineering, Shanmuganathan Engineering College, Pudhukottai, India \\ Email: prithivipranav@gmail.com,kkr_510@yahoo.co.in,vinobala186@gmail.com
}

How to cite this paper: Prithiviraj, A., Krishnamoorthy, K. and Vinothini, B. (2016) Fuzzy Logic Based Decision Making Algorithm to Optimize the Handover Performance in HetNets. Circuits and Systems, 7, 3756-3777.

http://dx.doi.org/10.4236/cs.2016.711315

Received: May 2, 2016

Accepted: May 15, 2016

Published: September 16, 2016

Copyright $\odot 2016$ by authors and Scientific Research Publishing Inc. This work is licensed under the Creative Commons Attribution International License (CC BY 4.0).

http://creativecommons.org/licenses/by/4.0/

\begin{abstract}
A prominent growth in a wireless communication system provides a wide range of services for a heterogeneous wireless network thus accomplishing user's needs. These real time services are delay sensitive which requires a continuous internet connection. Assuring a seamless end-end connectivity without link outages at the mid of an action is a crucial ongoing problem. Thus the main objective relies on designing a cognitive algorithm to achieve the practical impossibility of having a sustainable net connection while roaming across various technologies. Hence, to fulfill this and degrade vertical handover (VHO) issues an enhanced fuzzy logic based algorithm is proposed. Further, it focuses on improving the transmission quality with low handover latency, less packet loss and reduced false handover. Along with this the algorithm achieves an accurate prediction in the decision making of an optimal network to which the idle node gets connected. The networking environment also has an efficient mobility management, where the functioning network controls the mobility of concerned mobile nodes. Finally the simulation process of this proposal on a comprehensive test-bed infers the fuzzy logic based algorithm, guaranteed seamless connection. Further, the system has a $1.6 \%$ improvement in reducing the handover delay and $1.3 \%$ in packet loss reduction than the existing approaches, which in turn improves the transmission quality.
\end{abstract}

\section{Keywords}

Seamless Connection, Vertical Handover (VHO), Fuzzy Logic, False Handover

\section{Introduction}

Current trends in wireless system, paved way for immense improvements in communication system. This facilitates on-going calls and services without any interruption 
during handover between heterogeneous networks. The environment is made compatible for these varying wireless technologies to provide continuous connection. Heterogeneous wireless networks are defined as the system with different radio access technologies like Global System for Mobile Communications (GSM), Wireless Local Area Network (WLAN), Universal Mobile Telecommunications System (UMTS), Wireless Fidelity (Wi-Fi) and Worldwide Interoperability for Microwave Access (WI-MAX). Handover is the process of Mobile Node (MN) moving from one wireless technology to another [1]. A mobile node that switches their connections during mobility with different networks having different characteristics is known as Vertical handover (VHO). This leads to lots of issues in mobility management, Quality of Service (QoS) support and seamless handover. The handover process among the same network or same radio technology is known as Horizontal Handover (HHO). The differentiation between VHO and HHO is represented in Figure 1. By ensuring seamless connection, the mobile node can transparently switch back and forth among different technologies, such that data transfer and communications are lossless without any crucial disrupt. Thus the real time delay sensitive services like audio, video and all multimedia communications achieve a considerable improvement in transmission quality.

Seamless handover [2] is accomplished by having a continuous connectivity, low handover latency and minimal packet loss. The time delay between the last data packet received from the old serving network and the first data packet received at the new serving network by the MN is defined as handover latency. Generally, it is the time required for $\mathrm{MN}$ to get connected into the new network. Handover latency is a critical issue for real time applications with mobility $\mathrm{MN}$ that needs to meet a required QoS. Packet loss is the amount of packets that are lost or corrupted such that it cannot reach the mobile node.

False handover is an act of bouncing between the home network and candidate network with a fallacious decision of optimal network. In establishing a new connection

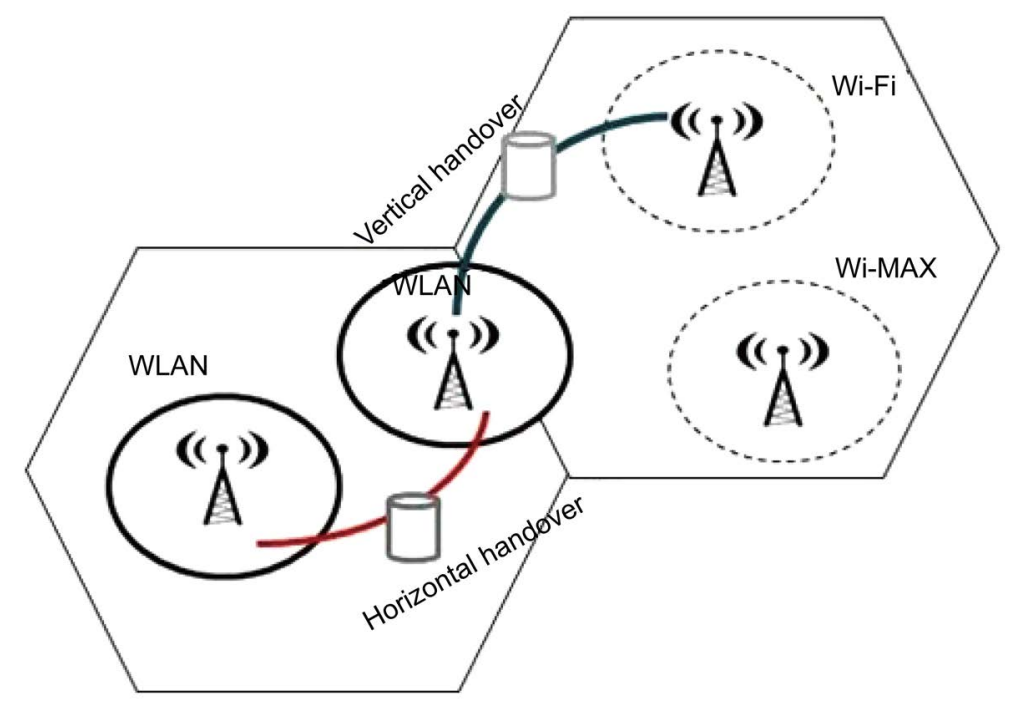

Figure 1. Horizontal versus vertical handover. 
using soft handover aids in reducing the delay and packet loss. Soft handover [3] utilizes make-before-break where a new connection is established with the optimal network before disconnecting from old network. In case of hard handover, a novel connection is done after the break of existing connection (break-before make).

Vertical handover includes three modules in its process: handover initiation, handover decision and handover execution [4] as shown in Figure 2. These phases take a sequential action; first the need of handover is identified by using different indicators called parameters. A standard threshold for each of these parameters is assigned and an indication is made when the measured values cross it. This phase is like a preprocessor of decision phase because the data required for decision making are gathered here.

Different parameters are clustered depending on their characteristics in defining system conditions like network-based, terminal-based and service-based [5]. Networkbased parameters are received signal strength, bandwidth, security and error rate. For a mobile node, a periodical measurement of RSS between MN and home network infers the availability of moving node. Similarly RSS of neighbor networks with MN is measured for predicting purposes. Difference in bandwidth availability has a direct effect on QoS during vertical handover. Error rate defines the ability of varying networks to be compatible for ongoing services. A secured data transfer is an important aspect of improving transmission quality. Terminal based parameters are velocity that determines mobility patterns of $\mathrm{MN}$ and power consumption. Service-based parameter states latency, reliability and data transfer rates of the provided services. Mostly all these parameters are highly correlated and cannot be expressed separately. In the process of achieving a seamless connection, using multiple parameters is highly preferable. This also improves the accuracy of decisions taken during handover.

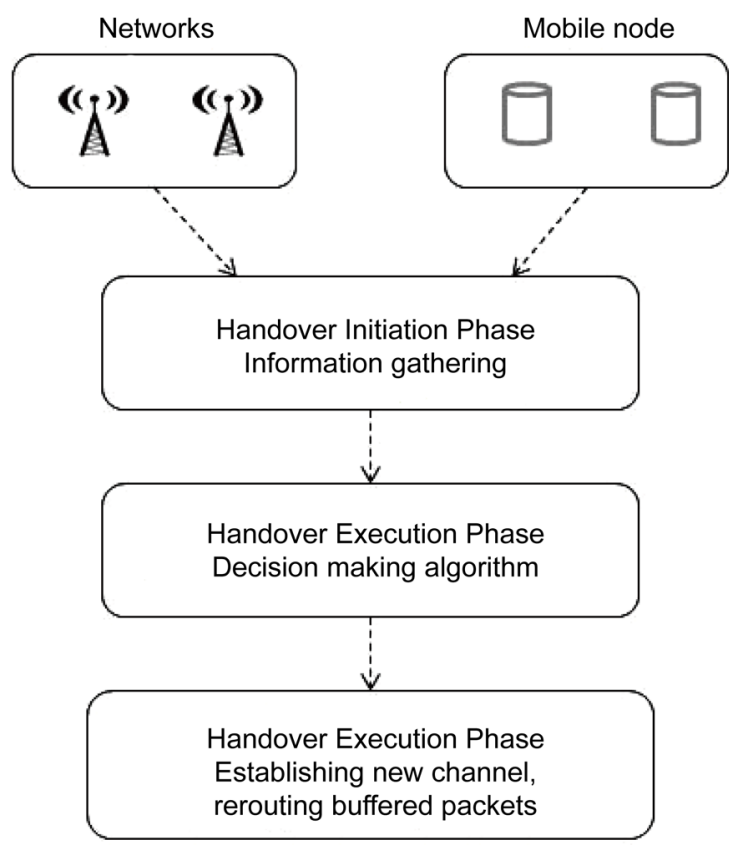

Figure 2. Phases of vertical handover. 
During handover decision phase, the available candidate networks in that environment, to which the MN can get connected, are evaluated. The outcome of this phase is a prediction of the best candidate network from the information gathered in the first phase. The algorithm should result in low handover latency, less packet loss and a reduced false handover. There are several decision making algorithms [6] used for vertical handover, some of familiar algorithms are function based, user centric, multiple attributes, markov based and fuzzy logic based. Among this entire algorithm, the fuzzy logic deals with uncertain constraints of real time values. Hence it results in a logical rule formulation. Sometimes a combination of fuzzy logic and multiple attribute works the best in a precise decision making. Other algorithms have implementation complexity, non-real time support and other service issues.

The last phase of vertical handover is handover execution where the transfer is exchanged to new network from old one. Handover should be executed considering implementation issues, such that existing infrastructure is less affected. A double buffer mechanism [7] is used to constantly reduce the packet loss. This avoids the out of sequence problem, for which it has two buffers forwarding packet buffer (FPB) and new packet buffer (NPB). FPB buffers and forwards the packet from the correspondent node to the home network. After a new channel is built with new network NPB buffers the packet of CN. A mobility management protocol [8] ensures continuity of the ongoing connections during the change of point of attachment by proactively determining the location of every mobile node in motion before the service is delivered. Thus a mobility management includes location management and handover management.

The proposed system model focuses the issues at all this phases and concludes with better solutions to it. The system uses the combination of all the features to improve the system performance like mobility management to ensure seamless connection, a fuzzy logical decision making algorithm for an accurate decision of handover network. Double buffer mechanism rectifies the packet loss and out-of-sequence problem and pre-detecting the nodes attachment through agents for reducing the handover delay by reducing the authentication delay. The rest of this paper has several sections which are dealing with background and related work in Section 2, architecture model of the system and workflow of the decision making algorithm is explained in Section 3, discussing the results and comparison in Section 4, the conclusion of the proposal and abstract plan over of extending the work in Section 5.

\section{Background and Related Work}

The existing algorithms on vertical handover decide the handover of mobile node, only based on received signal strength. Using a single parameter to finalize the handover process is not an efficient decision making algorithm. Nevertheless, using multiple parameters on handover initiation and deciding the best network, results in accurate handover detection. Distance is used to calculate how away the mobile node from the serving network and speed to measure the movement of MN. Fuzzy logic is one of the effective algorithms for decision making with more than one parameter. Some logical 
rules are formulated with choosing parameters and with which the priority of action to proceed is identified.

From the variant fuzzy engines [9] designed, the system achieves an accurate handover decision making of an optimal network. The normalization engine includes NQ, RS and DS fuzzy engines. Normalized values of data rate, latency and packet loss are processed in NQ and that generates the output score ( $Q$ value) that defines the quality of each candidate network. Similarly RS process normalized values of RSS and security ( $R$ value) and DS evaluates these two values to conclude with the final score. Ranking of candidate network takes place based on this score. By using the membership function, the real values of input parameters are converted into crisp values (low, medium, high) and with that fuzzy rules are generated.

Mamdani Fuzzy Interference System (FIS), which is an expert in logical knowledge, is used to extract the rules. The output crisp value is evaluated from the aggregated values of input parameters. The simulation results of fuzzy II, when compared to SAW, AHP and fuzzy I, shows an improvement in the case of video streaming. Further the work is enhanced by incorporating an adaptive normalization process for dynamically changing parameter's value. Decision parameters processing (DPP) module is included in the system which returns the current value of input parameters by communicating with the environment networks. The enhanced fuzzy III has a potential improvement in comparison with the fuzzy II on selecting the optimal network. Since the tradeoff of the working system accomplishes low satisfactory and existence of false handover is not much noted.

Handover delay during L2 connection with MIPV6 environment is addressed and rectified by fuzzy logic based mechanism [10]. Here the decision parameters are RSSI and speed, whose values are given as an input to the fuzzy inference system. By using GPS system speed of MN is monitored. Handoff latency is reduced by reducing delay during scanning, re-association and authentication. Thus the simulated results of handover between Wi-Fi, Wi-MAX and GSM have a reduced scanning delay.

An intelligent handover combining fuzzy logic and multiple attributes [11] ensures service connectivity by authenticating and registering into new network before connection breaks. By using context aware method, it collects all the information about decision parameters RSS, QoS parameters, battery status and velocity. This information is passed as inputs to fuzzy logic algorithm which decides the best handover network. Thus the system has an improvement in blocking probability, call drop, handover delay and less packet loss.

The environment is modeled to provide a seamless connection across heterogeneous network by using neuro-fuzzy multi parameter based vertical handoff decision algorithm [12] (VHDA). This uses six decision parameters RSS, velocity, number of users, bandwidth, coverage area and battery. The measured values of these decision parameters are converted into crisp values, incorporating from membership functions. A neural training methodology is used which trains the input output sets at each pass to membership function and error measure extracts the actual value by normalizing the 
real measured values. The simulation is done by changing number of handoff, path exponents, simulation time and distance among traditional, existing and proposed fuzzy method. This illustration results in an improvement in ping-pong effect, ESA and throughput.

\section{Proposed Methodology}

\subsection{System Model}

The proposed work deals with an intelligent computational algorithm on reaching an optimal solution to provide a promising seamless connection. The architecture model has a heterogeneous environment that portraits the handover between WLAN, Wi-Fi, WI-MAX and Cellular network as shown in Figure 3. The algorithms were designed in such a way; it can handle a possible number of parameters with reduced computation time. Here in this proposal, we focus on layer 2 handover process and their issues. The designed model has a decider hub, which is considered as the spine of the system. This acts as a centralized server where handover decision making and packets buffering takes place when the $\mathrm{MN}$ is idle. The heterogeneous network in the architectural environment is registered in decider hub. Each network has few agents (Aa) depend upon the coverage area which monitor about the ongoing status of node's mobility.

\subsection{Handover Initiation Phase}

Initially mobile node is connected to the MAG of home network, called HSMAG through which the communication between $\mathrm{MN}$ and correspondent node $(\mathrm{CN})$ takes

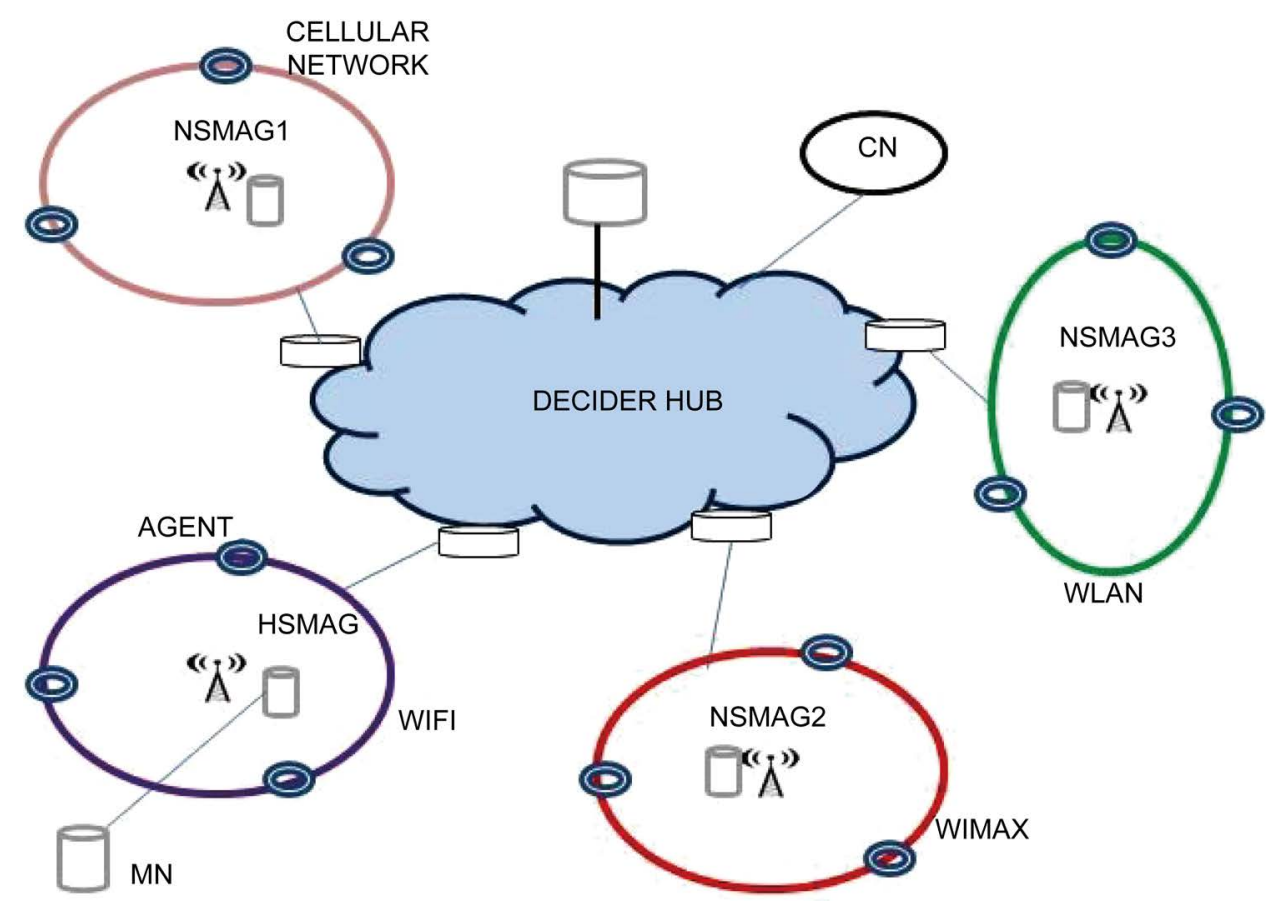

Figure 3. Proposed system model. 
place. Furthermore MAG of the network is in charge of authentication of mobile node entering in its coverage. The prefixed agents of the network monitor the movement of existing node and new arriving nodes, which orderly intimate to MAG and decider hub (DH). Agents are fixed in such a way that it can cover the action of a node in the coverage zone because of that the number of agents differs with the network. The DH on receiving these factors rearranges its routing table for achieving exact transmission to the destination network. While monitoring $\mathrm{MN}$, the agent in prior recognize about the disconnection of a mobile node from home network. This action is predicted from the periodical measurement of RSS and distance values between moving node and fixed network. Signal strength degrades when the MN moves away; similar to this distance is far away. These two parameter value intimate the $\mathrm{DH}$ about handover initiation. $\mathrm{DH}$ in turn broadcast about the idle state of $\mathrm{MN}$ to all other networks in the environment along with MN profile. Thus the authentication delay is reduced as the neighbor network previously has $\mathrm{MN}$ profile. The values of initiator parameters are also measured between moving $\mathrm{MN}$ and neighbor networks. Along with this, velocity and location of node is measured. All the gathered information is utilized by the decision phase for the accurate prediction of the new network.

\subsection{Handover Decision Phase}

The MN is isolated in the environment being disconnected from a network and it is idle without any transmission and communication. In progress of achieving seamless connection MN needs to get connected to any of the candidate network which best suits the ongoing service. The decider hub is embedded with the decision making algorithm and the inputs to this algorithm are the information gathered in the first phase. DH is a centralized server which controls over all the action on the environment. The correspondent node's data transfer to the destination node takes place via $\mathrm{DH}$. This hub routes the packet to exact destination network MAG to which the MN is connected. Varying networks, agents and mobile nodes in system environment get registered with their profile in $\mathrm{DH}$. The proposed system adopts fuzzy logic based algorithm for deciding the suitable handover network.

\section{Fuzzy Interference System}

A fuzzy logic based system experts in an accurate decision making of an optimal network. Zero-order Sugeno Fuzzy inference system [13] works best in logical rule formation and decision making. FIS takes the benefits from set theory to map input and output values from the computed input values.

The first step of FIS is to generate fuzzy rules from the collection of linguistic values. Fuzzy rules are in an if... and/or... then format. The membership function outlines the linguistic values for every measured real value of input parameters. This process of taking input values and converting them into linguistic values is fuzzification. A combination of trapezoidal and triangular membership function are used to extract more rules, thus to improve the prediction accuracy. The input parameter values of RSS, dis- 
tance and speed values are measured for every neighboring network with that of the mobile node in a periodical session. $\mathrm{R}_{\mathrm{s}}$, set of signal strength values of all networks, similarly $\mathrm{D}_{\mathrm{s}}$, set of measured distance value and $\mathrm{S}_{\mathrm{d}}$ is the current speed of MN.

The linguistic values are inferred from the membership function [14] with that of normalized value as shown in Figures 4-7. Here a combination of triangular and trapezoidal membership functions with overlap is used. The real values are mapped to linguistic values depending on their range mentioned in membership function. The linguistic values of RSS are low, medium, high, for velocity slower, slow, average, fast, faster and for distance short, near, medium, far and away. A different combination of these parameter values is expressed in fuzzy rule from which the decision is made.

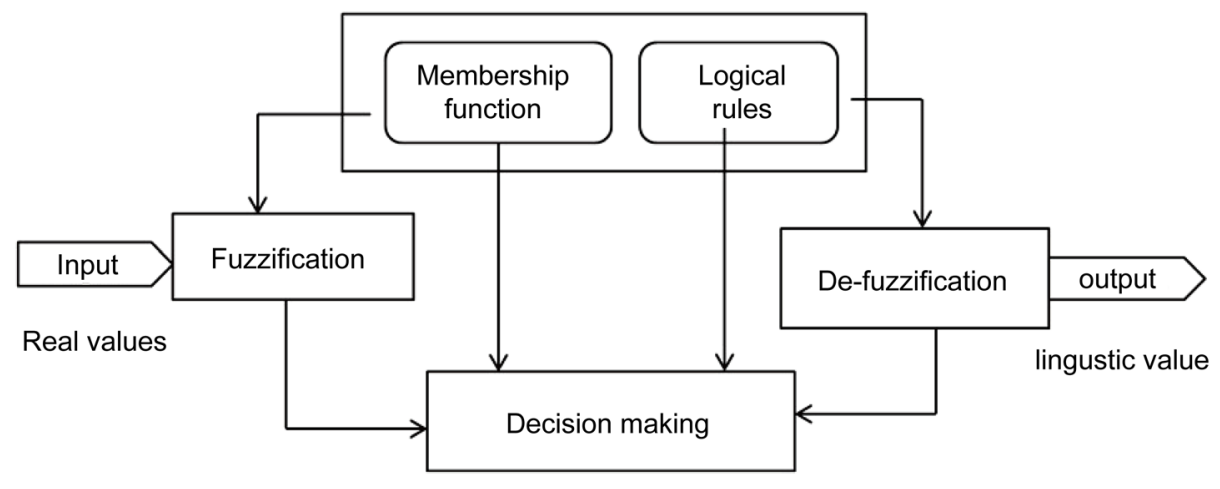

Figure 4. Fuzzy inference systems.

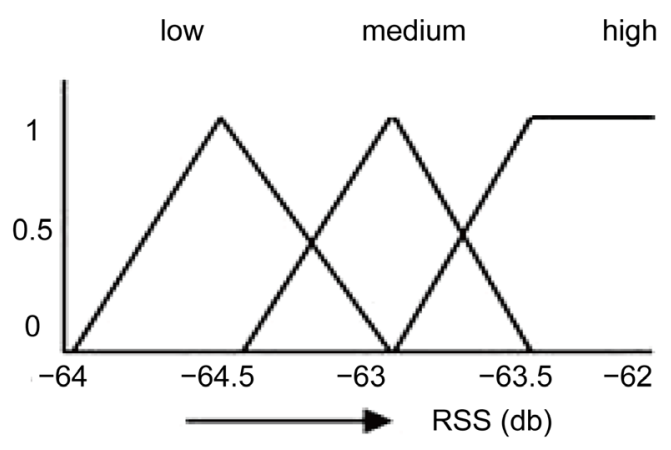

Figure 5. RSS-membership function.

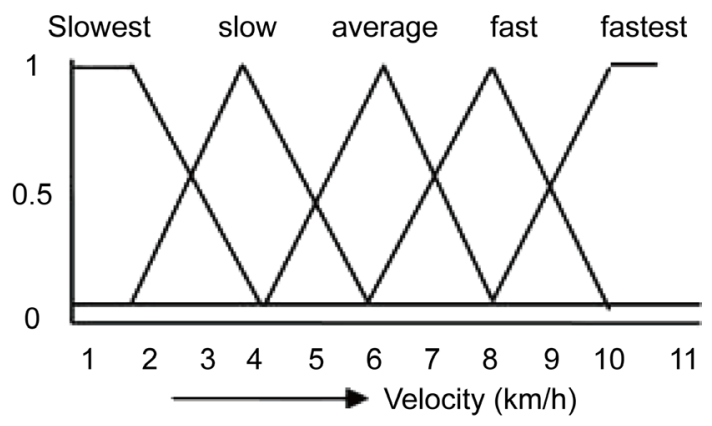

Figure 6. Velocity-membership function. 


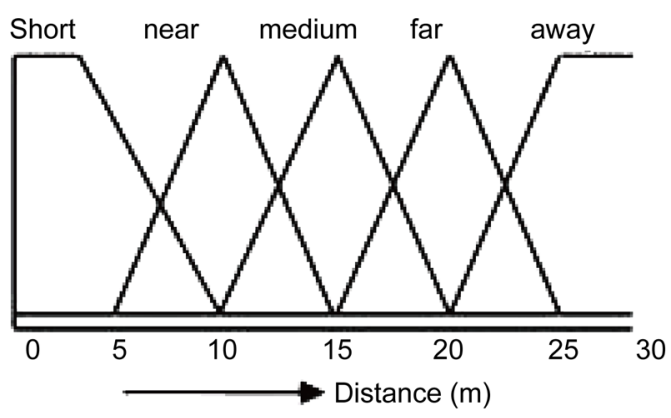

Figure 7. Distance-membership function.

The next step is evaluating rule strength by combining the fuzzified values in accordance with fuzzy rules. Fuzzy combination operators are "and" and "or" that are represented as T-norm. On using "and" rule strength bags a minimum value from the set of fuzzified values. In case of "or" it holds maximum value. $\mu$ RSS(x) results with network having minimum value received signal strength at a particular time $\mathrm{x}$ which was shown in Equation (1). Likewise node close to any of the candidate network is identified from $\mu \mathrm{DS}(\mathrm{x})$ represented in Equation (2)

$$
\begin{aligned}
& \mu \operatorname{RSS}(\mathrm{x})=\mathrm{T}-\operatorname{norm}[\min \mathrm{x}[\mu \operatorname{Rs}(\mathrm{i})]], \text { for } \mathrm{i}=1,2, \cdots, 5 \\
& \mu \mathrm{DS}(\mathrm{x})=\mathrm{T}-\operatorname{norm}[\min \mathrm{x}[\mu \operatorname{Ds}(\mathrm{i})]], \text { for } \mathrm{i}=1,2, \cdots, 5
\end{aligned}
$$

$\mathrm{SD}(\mathrm{x})$ holds the speed of the moving node at the time $\mathrm{x}$, with this the chance of attachment to nearby network is guessed. The formulated logical fuzzy rule base is represented in the Table 1.

Finally defuzzification [15] results in a linguistic output for each rule which is then prioritized to make an accurate decision. The output of the system is a singleton spike extracted from the constructed logical rules

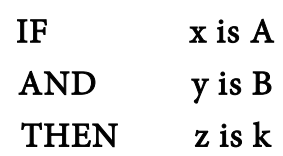

where $\mathrm{k}$ is a constant output of fuzzy rule inferred from the consequent membership function as shown in Table 1. The fuzzy output implies the network to which MN is close enough and clues to predict the optimal network.

\subsection{Handover Execution Phase}

The network, which has high priority of getting connected to $\mathrm{MN}$, is predicted by decision phase. In accordance with this prediction a new communication channel is built between MN and NSMAG. As the predicted handover network has an MN's profile, the authentication delay is reduced considerably. Decider hub modifies its routing table information about the change in the new destination network to which the data of $\mathrm{CN}$ should be transmitted. After successful establishment of an optimal route to an optimal network, data transfer takes place. Before that, the buffered packets which should be reached to $\mathrm{MN}$ during its idle state is rerouted to its new connection without any loss. 
Table 1. Fuzzy rules.

\begin{tabular}{cccc}
\hline & IF & & THEN \\
\hline RSS & Velocity & Distance & Handover \\
Low & Slowest & Away & Low \\
Low & Slow & Away & Low \\
Low & Medium & Far & Medium \\
Medium & Slow & Far & Medium \\
Medium & Average & Medium & High \\
Medium & Fast & Far & Medium \\
High & Fast & Medium & Low \\
High & Average & Near & Medium \\
High & Slow & Medium & Low \\
Low & Fast & Near & High \\
Low & Medium & Short & High \\
Medium & Slow & Near & High \\
Medium & Average & Medium & Medium \\
\hline
\end{tabular}

Execution phase of handover also takes care of mobility management for providing effective seamless service utilization. Here the network in connection to MN manages the mobile node, where the $\mathrm{MN}$ is free from mobility concerned issues.

The pseudo-code of the proposed algorithm depicts the work flow.

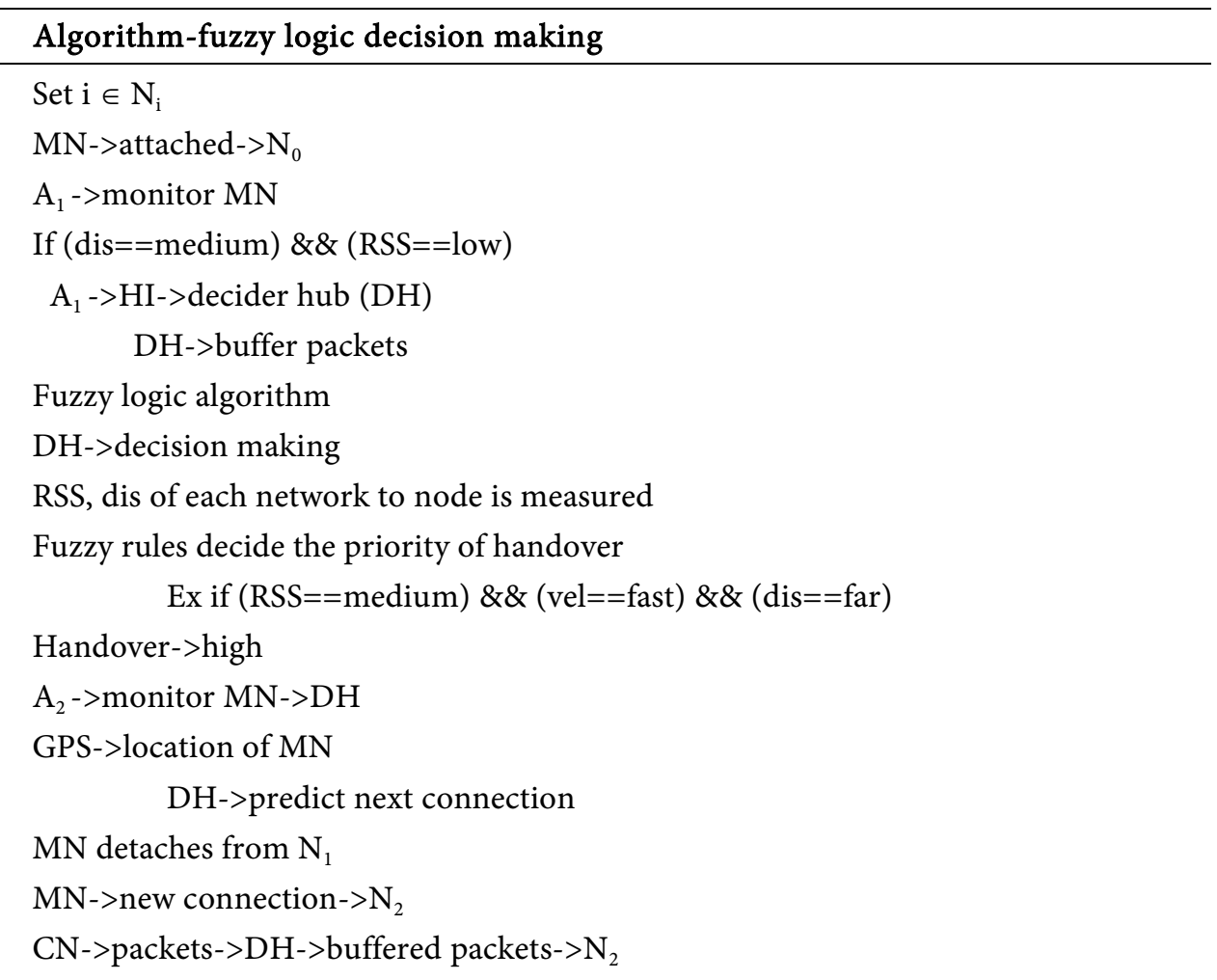


The working flow of the proposed system model is shown in Figure 8.

\subsubsection{Message Signaling Flow}

The step by step procedure of the proposed system as represented in Figure 9, works as follows,

Step 1: The mobile node gets attached to a network to access the services and applications exist in internet globally. If a node wishes to get connected to a network, MAG of network authenticates the MN and assigns an ID to it. Then MAG sends a proxy bind update message (PBUM) to $\mathrm{DH}$ along with the MN's profile. By this $\mathrm{MN}$ gets registered in the server for identifying the destination route.

Step 2: The DH response with a proxy acknowledgement message (PBAM), thus a transmission channel is built between them. For the mobile node, the attached network becomes the home network (HN) and MAG as home serving MAG (HSMAG).

Step3: CN communicates with MN via DH and HSMAG. The routing table of $\mathrm{DH}$ guides the packet transfer to reach the correct destination network. The data packet is prefixed with MN-ID, by this the HSMAG identify the destination node that waits for data. Thus a successful data transmission occurs between $\mathrm{MN}$ and $\mathrm{CN}$.

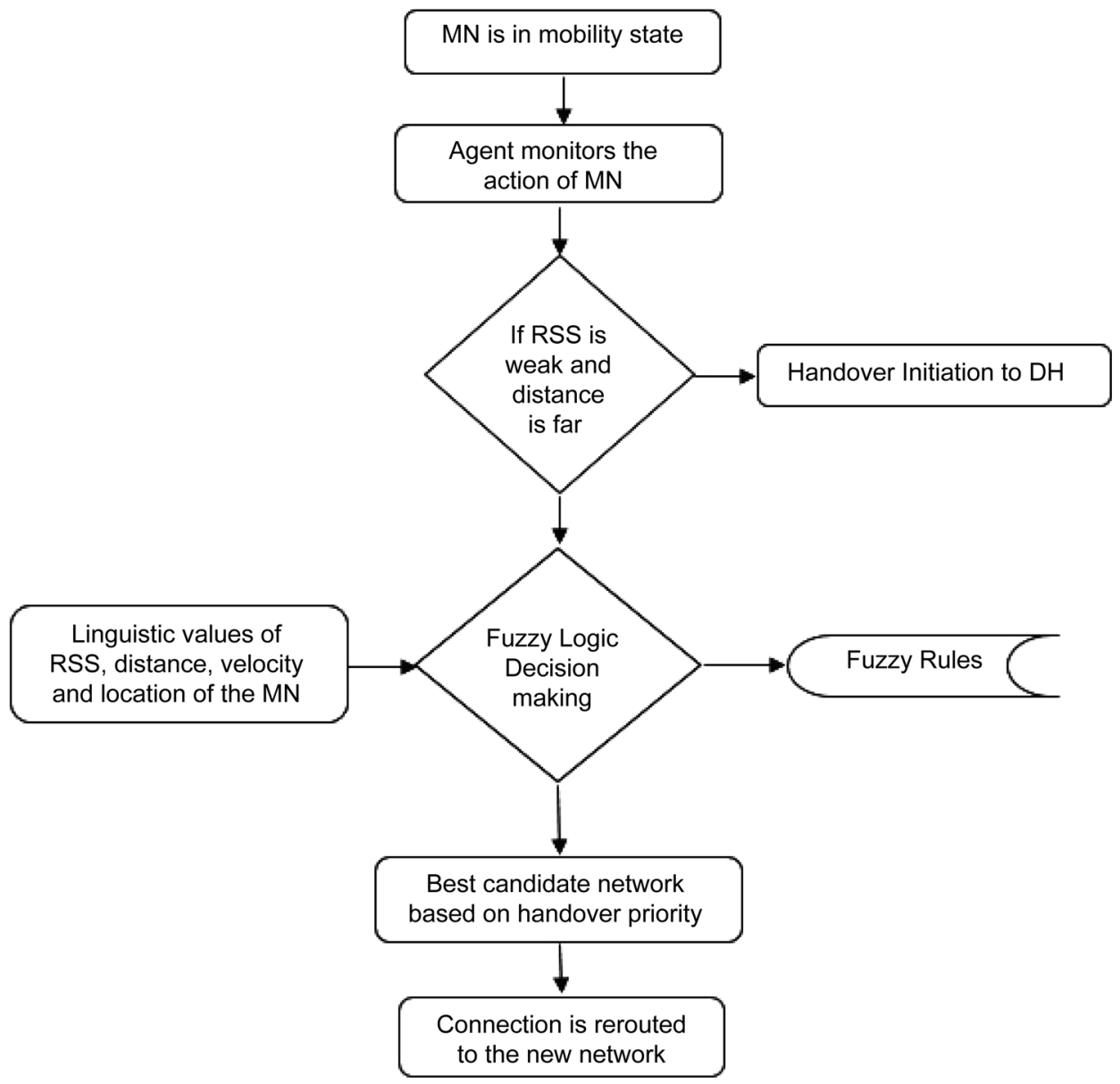

Figure 8. Working flow of the proposed model. 


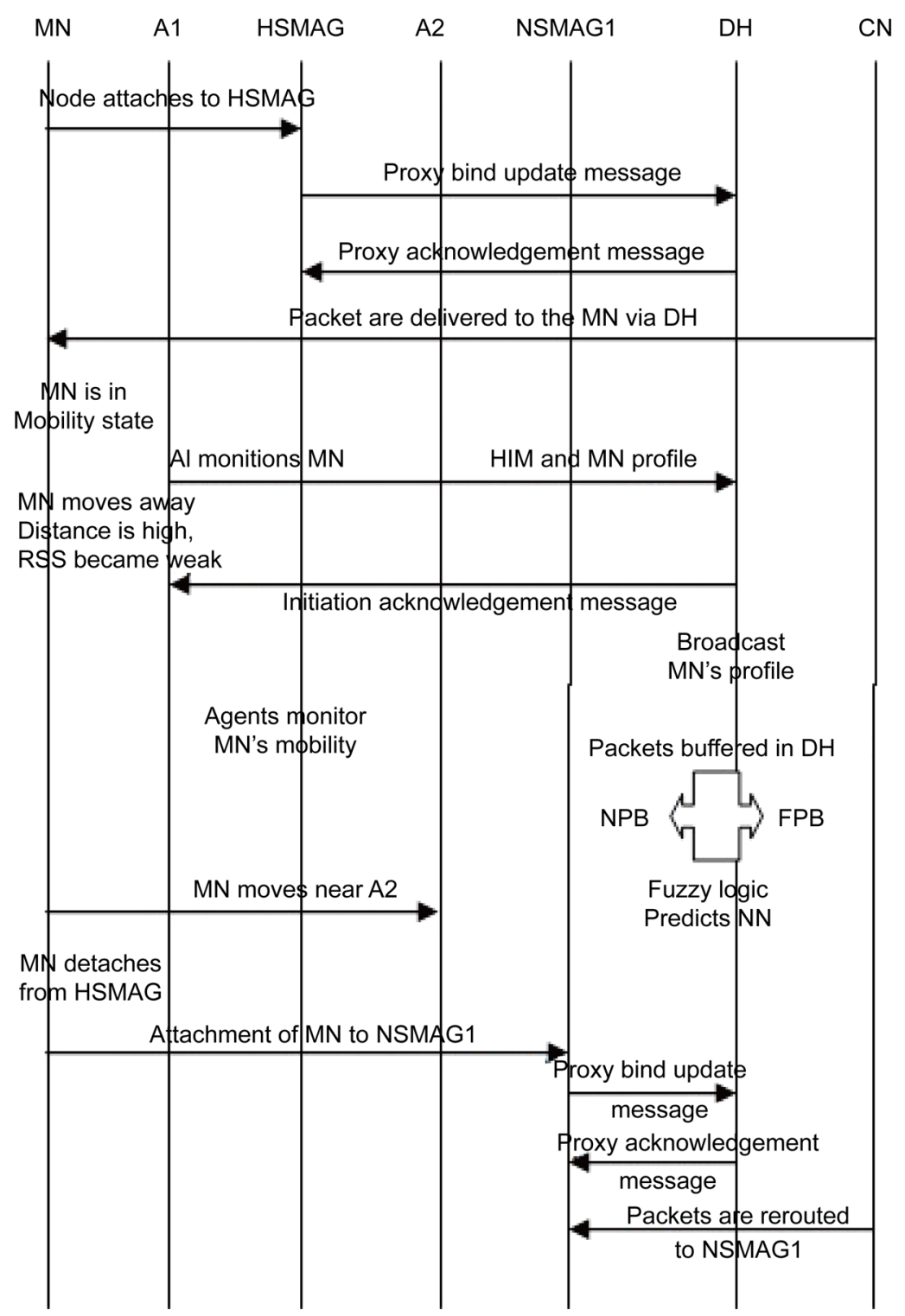

Figure 9. Message signal flow of the proposed system.

Step 4: As the $\mathrm{MN}$ is in mobility state it keeps on moving away from $\mathrm{HN}$, this results in detaching from $\mathrm{HN}$.

Step 5: Before the exact detachment happens, the agents fixed at end of coverage area monitor the movement of MN. By analyzing the RSS and distance value between $\mathrm{HN}$ and $\mathrm{MN}$, the Aa intimates the MAG which in turn intimates the DH about the disconnection to happen.

Step 6: Handover initiation message (HIM) is sent to DH along with the MN's profile. DH replies back with an initiation acknowledgement message (IAM).

Step 7: Decider hub, broadcast the MN's profile to the entire neighborhood network in the environment. Thus, by this candidate network awaits for an isolated node to get attached to it. 
Step 8: Additionally, on receiving HIM, DH starts to buffer the packets received from $\mathrm{CN}$ by using the double buffer mechanism as in Section 3.4.2, to avoid packet loss when the MN enters handover session.

Step 9: Meanwhile MN completely gets disconnected from $\mathrm{HN}$ and enters into handover session. The communication channel is broken between the isolates node and $\mathrm{CN}$.

Step 10: At this stage DH overlooks the movement of $M N$ and infer the location of MN periodically. Fuzzy logic based decision algorithm embedded in DH starts to predict the MN's new attachment network (NAN).

Step 11: The agents of other networks like Ab start to monitor the mobility of $M N$ and measures RSS, distance and velocity value between isolated MN and the corresponding network. These values are then sent to $\mathrm{DH}$ for deciding the best network to which $\mathrm{MN}$ can get connected. Decision making algorithm works as explained in prior Section 3.3.

Step 12: On a successful prediction, the NSMAG of NAN authenticates the idle node. As mentioned in Step 1, 2 and 3, a communication channel is built between MN and $\mathrm{CN}$ via $\mathrm{DH}$ and NSMAG.

Step 13: DH routing table is modified with the route for NAN for accomplishing an exact data transfer between $\mathrm{MN}$ and $\mathrm{CN}$.

Step 14: After establishing an optimal route. DH starts to transmit the buffered packets till that time. By means of double buffer mechanism, the packets reach the destination node in a correct sequence. Thus packet loss is reduced to a great extent.

Step 15: This is layer 2 connections, likewise the next detachment and attachment is a layer 3 connections.

\subsubsection{Buffering Mechanism}

Once, after disconnecting from the home network there were no data transfer between $\mathrm{MN}$ and $\mathrm{CN}$. At this stage data packet of $\mathrm{CN}$ fails to reach the destination mobile node. Until a best candidate network is found and new connection made these packets are buffered in decider hub. Later, when a new channel is established, the buffered packets are re-transmitted to the new network. Buffering mechanism on retransmission of buffered packets misses the packet sequence. In order to avoid this sequence problem, a double buffer mechanism [7] is used. This buffering method has two buffers: forward packet buffer (FPB) and new packet buffer (NPB). The buffering process is explained in below steps

Step 1: CN keeps on transmitting the data packets to the home network via decider hub. When $\mathrm{DH}$ receives the handover initiation message, it will intimate FPB to buffer and forward the packets to HSMAG.

Step 2: When MN gets disconnected from home network, DH intimate the NPB to save the sequence number at the time of disconnection happened. It also buffers and stores the packets from that sequence number.

Step 3: DH checks the sequence number and place it in their corresponding buffers.

Step 4: As soon as a new connection is established, packets in FPB is transmitted in 
advance to packets in NPB. By this MN receives the packet in sequence without a loss. Prior transmission of FPB results in duplication of packet which is depleted at the destination end.

\subsubsection{False Handover}

False handover is an unsuitable prediction of handover results in switching the connection between other candidate networks within a short span. The MN bounces their transmission link back and forth with more networks [16]. This leads to disruption in communication quality and delay in establishing a suitable transmission channel. Furthermore packet loss occurs when DH starts to send the buffered packets to a new network, but the MN switches their connection to another network. Thus the transferred packet does not reach the destination node due to false predictions. When the system fails have an accurate decision making algorithm, unnecessary handover happens because of the bad decision. Hence, fuzzy logic based algorithm reduces false handover by using the decision making parameters distance and speed. Determined by the membership function, if the speed of $\mathrm{MN}$ is faster and faster and distance is average and far, there is a chance of false handover to occur. This infers that $\mathrm{MN}$ is moving with a great speed and it won't maintain a stable connection for a long time. By this the Ping-Pong effect of MN and packet loss is reduced, resulting in an improved transmission quality.

The signal flow in Figure 10 depicts the occurrence of false handover where the unnecessary handover resulted in a loss of transmitted packets and further increase the handover delay. Incorporating fuzzy based decision making algorithm as in Figure 11, pin point the handover network is a false handover from the decision making parameters distance and velocity. Thus the mobile node is aware of unnecessary handover and delay is reduced. Current location of the mobile node is identified and the direction of moving node is determined by a probability density function [17]

$$
f(\theta)=\frac{1}{2 \pi},-\pi<\theta<\pi
$$

where $\theta=\arctan \left(\frac{a}{2 D}\right)$, “ $a$ " is cell coverage and “ $D$ ” is the distance. When the MN's direction of movement is within $\theta \in(-\theta, \theta)$ and measure form SMAG to MN there is a need for handover, otherwise it is a false handover. Thus the probability of false handover initiation is

$$
P_{a}=1-\int_{-\theta}^{\theta} f(\theta) \mathrm{d} \theta
$$

\footnotetext{
Algorithm False handover

If $\mathrm{MN}$ within cell coverage

If (dis $==$ far) \& \& (vel==fast)

$\mathrm{MN}$ moves away with high speed

$\mathrm{MN} \neq>$ connectionN2

Unnecessary handover
} 


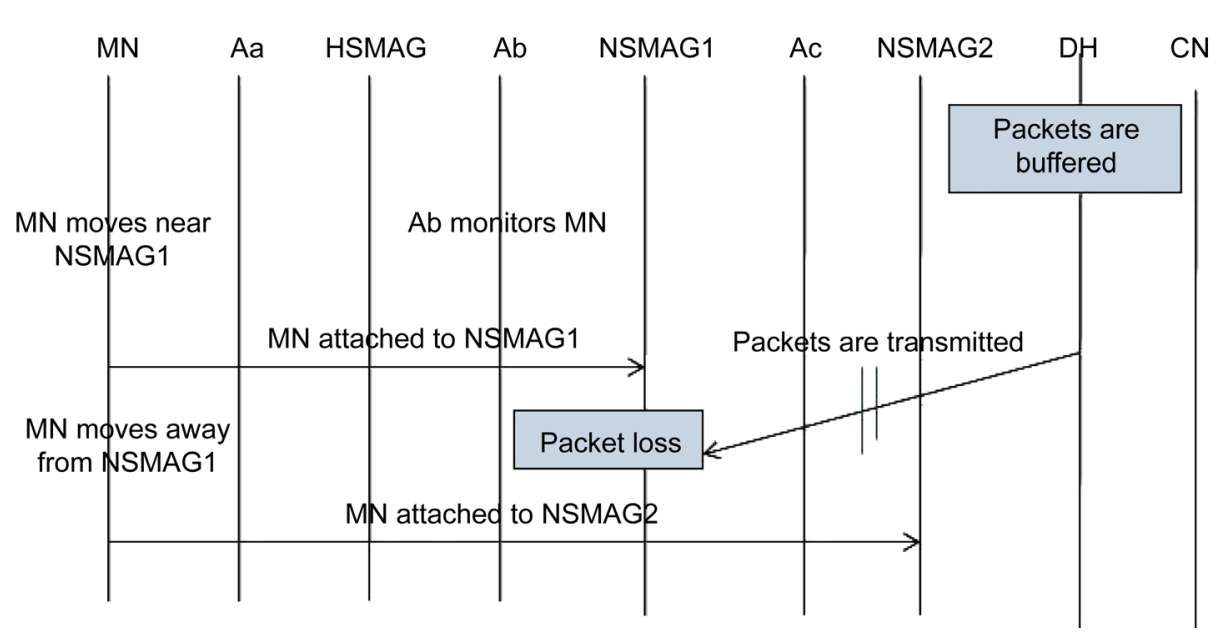

Figure 10. False handover.

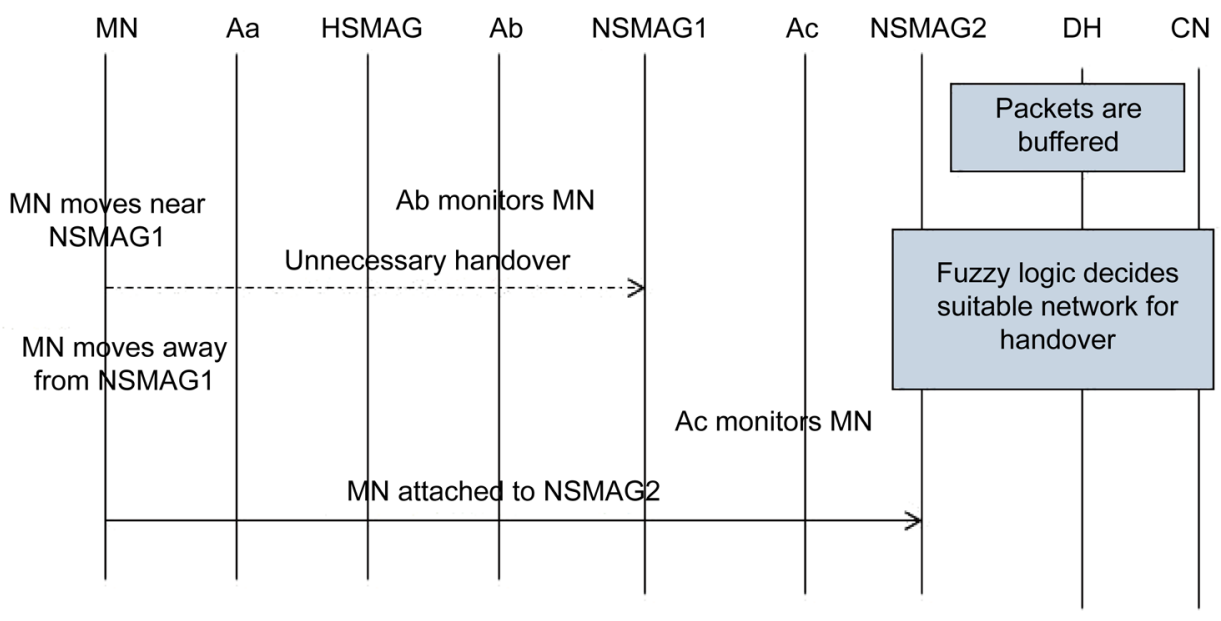

Figure 11. Reduction in unnecessary handover using fuzzy logic.

The working flow of the false handover algorithm is represented below in Figure 12.

\section{Results and Discussion}

The designed algorithm is simulated using NS-2.29 [18] simulator, NIST mobility module [19] along with the IEEE 802.21 standard framework called Media independent handover (MIH) [20]. This framework enables seamless continuity during MN movement between heterogeneous networks. It has additional functionality called, media independent handover function with three services, media independent event service (MIES), command services (MICS) and information services (MIIS). These services are responsible for ensuring handover transparency, detecting events and providing information about networks, node movement to handover server. The system model is simulated in a test bed equipped with MN, Wi-Fi, Wi-MAX [21], cellular network, WLAN, CN and decider hub which act as a centralized server. DH is in charge of monitoring the overall activity of the nodes in the test bed. The networks and mobile nodes 
get registered in the hub once it entered into that environment, that's making it easy to track the movement. The networks are configured with their respective network patch file compatible for the simulator. The mobile nodes and networks are configured in such a way that the mobility management of moving node is controlled by connecting network. This makes the MN unaware about the handover and provides continuous service utilization. Message delivery takes place between $\mathrm{MN}$ and $\mathrm{CN}$ routed by the server and the connected network. The delay is measured between the time, the transmission broken and the time at which the transmission resumed when connected a new network. This test bed configuration helps to understand a realistic working of layer 2 connectivity. The parameter specification of nodes used in the environment is explained in Table 2 [22] [23].

Depending upon the coverage of each network number of agents is placed proportionally to track the movement of $\mathrm{MN}$. The agents communicate the collected information to SMAG which in turn transmits to the hub. These agents helps in pre-judging the disconnection of MN by measuring signal strength, distance and speed of moving

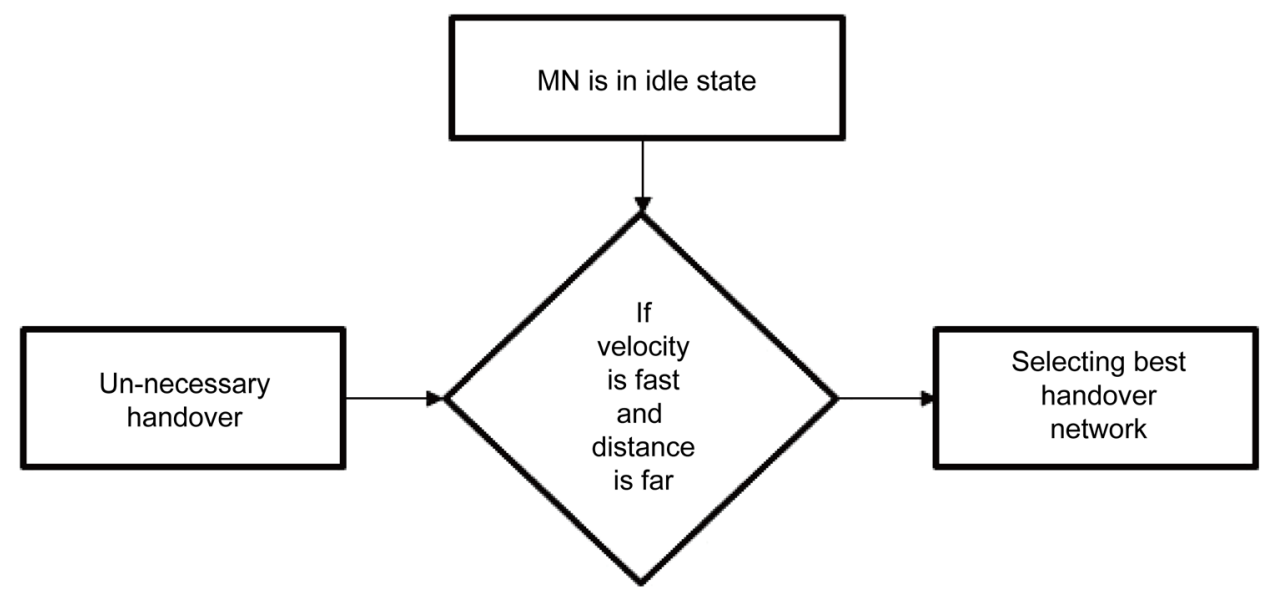

Figure 12. Working flow of the false handover algorithm.

Table 2. Simulation parameters.

\begin{tabular}{cc}
\hline Parameters & Values \\
\hline Simulation duration & $1,500 \mathrm{~s}$ \\
CBR packet size & 250 bytes \\
Number of CBR packets & 10,000 \\
Routing protocol & AODV \\
Mobile node speed & $1.5 \mathrm{~m} / \mathrm{s}$ \\
Data rate & $1 \mathrm{Mbps}$ \\
MN cache size & 2048 \\
Handover & Active \\
IEEE 802.21 maximum bandwidth & $10 \mathrm{Mbps}$ \\
\hline
\end{tabular}


node. The signal strength is calculated [10] using

$$
10 \log _{10} P_{r}=10 \log _{10} P_{0}-10 \alpha \log _{10} D+X
$$

where, $10 \log _{10} P_{0}=10 \log _{10} P_{t}+10 \log _{10} G_{t}+10 \log _{10} G_{r}+20 \log _{10} \frac{\lambda}{4 \pi}$ is the received power, $\lambda$ is the wavelength, $P_{t}$ is the transmission power and $G_{t}=G_{r}=1$ are the transmitter and receiver antenna gains respectively. $\alpha$ is the path-loss gradient. $X$ is a zero mean Gaussian random variable. The mobile nodes in the test bed are specified with a random motion and random speed in a periodical time. Thus the velocity depends on the magnitude and direction of movement and it is measured using [24].

$$
V_{i}=V_{i} * p+\sqrt{1-p^{2}} * V_{m} * X
$$

where $V_{i}$ is the complex speed of MNs, $p$ is the correlation of the velocity between time intervals. $X$ is Rayleigh distributed magnitude. $V_{m}$ is the mean speed of mobile nodes. Along with this the distance $D$ is measured between MN and SMAG. After handover, the distance calculated between $\mathrm{MN}$ and all neighbor networks. Similarly, signal strength is measured periodically between node and candidate networks. By using these parameter values the decision making of best handover network is predicted. These real time values are normalized by a normalization function [25].

$$
N=\frac{v a l-v a l_{\min }}{v a l_{\max }-v a l_{\min }}
$$

The Normalization function of signal strength is,

$$
N R S= \begin{cases}0, & 0 \leq R S \leq R S_{t h} \\ \frac{R S_{x}-R S_{t h}}{R S_{m}-R S_{t h}}, & R S S_{x}>R S_{t h}\end{cases}
$$

$R S_{x}$ is the measured values, $R S_{t h}$ is the threshold value assigned and $R S_{m}$ is the expected maximum values. Similarly, the normalization function of velocity is,

$$
N V= \begin{cases}0, & 0 \leq V_{x} \leq V_{m} \\ 1-\frac{V_{x}}{V_{m}}, & 0 \leq V_{x} \leq V_{m}\end{cases}
$$

The normalized function of distance is

$$
S= \begin{cases}0, & d \leq d_{\max } \\ \frac{d}{d_{\max }-d}, & 0 \leq d \leq d_{\max }\end{cases}
$$

The normalized values are then converted into linguistic values using the membership function as represented in Section 3.3. Here we have three parameters and three membership functions, thus the possible rule combinations are $33=27$ [26]. Each network in the environment takes these 27 rules for achieving an accurate decision making. From this different combination of rules, the priority of a node to be connected to that network is predicted. The output of the rule base is high, medium and low. For example, if the signal strength is medium, velocity is slow and the distance is far, the 
output is medium. This says there is average chance for node to get connected to that network. Similarly, if signal strength is high, velocity is average and distance is near there is high possibility for the node to get connected. Likewise the fuzzy logic algorithm using logical rule, decides the best handover network. Apart from these logical rules, the server also considers the current location of $\mathrm{MN}$ in the environment to make higher accuracy in deciding best network. By means of global positioning system (GPS) the MIIS server of MIH with a broadcast mechanism acquires the current location of the MN. For more specific output the coverage is parted into different zones [27], so when the node handover within the same zone the MIIS query is reduced as it has enough information already. From this the handover latency can be further reduced by reducing the query delay.

The handover latency is reduced by reducing the authentication delay, this done by the earlier prediction of nodes arrival by the agents. $\mathrm{RS}_{\min }(-65 \mathrm{db})$ and $\mathrm{RS}_{\max }(-60 \mathrm{db})$ is predefined, when the signal strength reaches $\mathrm{RS}_{\min }$, the agents intimate $\mathrm{DH}$, there is possible handover. And when it reaches $\mathrm{RS}_{\max }$, the communication is broken and the MN waits for its layer 2 connectivity. During L2 the agents of that network measure signal strength and if it reaches $\mathrm{RS}_{\max }$ it predicts a possible connection. As it already has $\mathrm{MN}$ profile broadcasted by $\mathrm{DH}$, the new network (NN) authenticate the node. So if it reaches $\mathrm{RS}_{\min }$ for that network the connection is established. By this, the delay of waiting for service and buffered packets is reduced. An illustration is made using real time scenarios in IEEE 802.21 to demonstrate the performance progress of the proposed algorithm with the existing one regarding delays and packet loss. During handover between Wi-Fi and Wi-MAX the data transmitted by CN is buffered in decider hub using and the connection establishment delay is reduced by reducing the authentication delay. Thus the video, audio and image streaming is improved by $7.75 \%$ better than traditional fuzzy (T.fuzzy) algorithm and the fuzzy I-based HDS [9] as compared with proposed fuzzy decision making (FDM) shown in graph Figure 13.

The packet loss during handover is reduced by buffering the packets in hub using double buffer mechanism. This also rectifies the out-of-sequence problem during

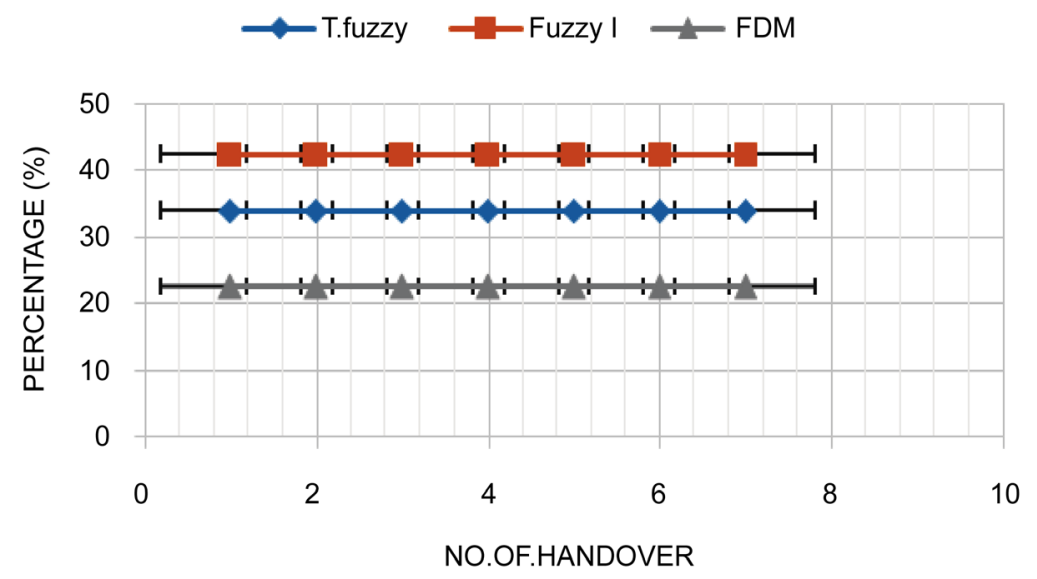

Figure 13. Performance improvement in streaming data packets. 
re-transmission of data to the new network. Thus on experimenting the proposed idea, the data packets from the corresponding node are set to be buffered in the server during mobile node's idle state. Until the handover network is identified, the packets are stored without letting any packet loss. Compared with the intelligent decision algorithm (IDA) [11], the packet loss is reduced as the server starts to buffer in FPB early at the handover initiation message. Upon the disconnection, the NPB saves the sequence number and also buffers the packet. Thus on using two buffers the packet loss is reduced considerably up to $1.3 \%$ in reduction of packet loss compared to the existing algorithm, as in Figure 14. Addition to this as NPB focus on saving the sequence number on re-transmission to the new network the packets will be transmitted in an exact sequence from where it is disconnected. Thus the proposed model addresses and rectifies the out-of-sequence problem.

The authentication delay is reduced by pre-detecting the MN's arrival via agent and authenticates it earlier. When the signal strength between the mobile node and the candidate network reaches $\mathrm{RS}_{\max }$ (predefined value), the agent of that network intimate the NSMAG about the arrival of node. Thus the network, authenticate the node by contacting the server and if it reaches $\mathrm{RS}_{\text {min }}$, the agents confirms the prediction of mobile node's attachment with it. So finally when the node is into networks coverage the communication channel is built without any delay for authenticating the node and this in turn reduces the overall handover delay. The accurate prediction of candidate network by fuzzy logic algorithm also helps in altering the routing table; hence the packets are rerouted as soon as the channel is built. All together, the simulated system has a much reduced delay of $1.6 \%$ than the fuzzy logic based scanning delay (fuzzy delay) [10] as shown in Figure 15.

\section{Conclusion and Future Work}

This paper addresses the issues in vertical handover and mobility management by proposing an intellectual algorithm to overcome these issues. This work increases the ability to continue accessing the ongoing services even when the node is handover to different network functionality. A seamless handover with low handover delay and packet loss is obtained by incorporating fuzzy based decision algorithm. The predefined rules

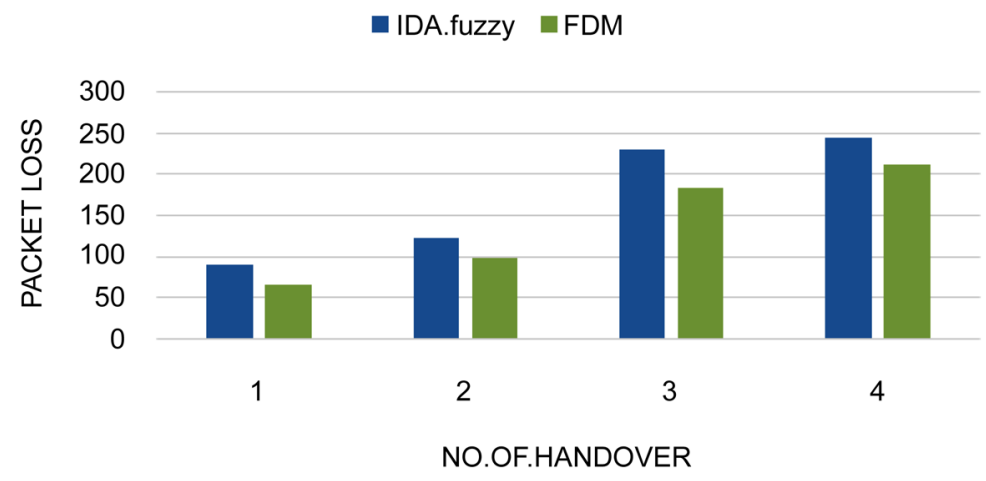

Figure 14. Reduced packet loss. 


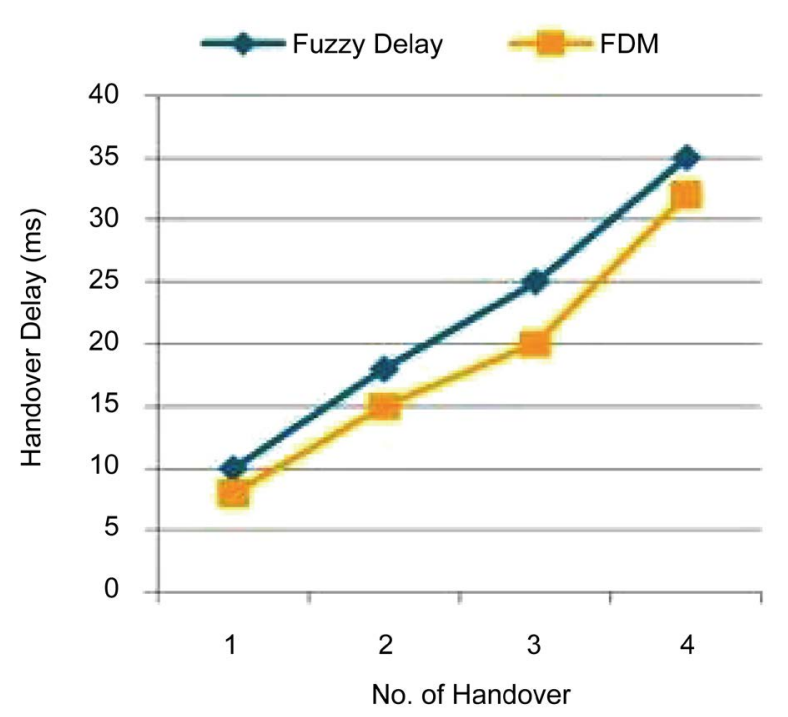

Figure 15. Reduction in handover delay.

of fuzzy decide the best network to which an isolated node can be connected by using decision parameters. Along with this the false handover probability of node getting connected to the network is estimated, which is much reduced in the proposed work. Furthermore a double buffering mechanism reduces packet loss by buffering data in two separate buffers. This also focuses in rectifying the sequence problem on retransmission of data. Thus, all together the proposal to keep up seamless connection had an improvement in reducing the delay up to $1.6 \%$ and packet loss to $1.3 \%$. Advancement to this, the algorithm is going to be tested in a PMIPV6 environment with more number of nodes taking successful handovers. Along with this, the work is further improved by deploying specific node locator other than GPS to achieve more accuracy.

\section{References}

[1] Jaraiz-Simon, M.D., Gomez-Pulido, J.A. and Vega-Rodriguez, M.A. (2015) Embedded Intelligence for Fast QoS-Based Vertical Handoff in Heterogeneous Wireless Access Networks. Pervasive and Mobile Computing, 19, 141-155.

http://dx.doi.org/10.1016/j.pmcj.2014.01.009

[2] Seamless Handover. http://www.cs.ucla.edu/classes/fall03/cs218/report/handoff.doc

[3] Yan, X., Y. Sekerciog, A. and Narayanan, S. (2010) A Survey of Vertical Handover Decision Algorithms in Fourth Generation Heterogeneous Wireless Networks. Computer Networks, 54, 1848-1863. http://dx.doi.org/10.1016/j.comnet.2010.02.006

[4] Zekri, M., Jouaber, B. and Zeghlache, D. (2012) A Review on Mobility Management and Vertical Handover Solutions over Heterogeneous Wireless Networks. Computer Communications, 35, 2055-2068. http://dx.doi.org/10.1016/j.comcom.2012.07.011

[5] Chandavarkara, B.R. and Rammohan Reddy, B. (2012) Survey Paper: Mobility Management in Heterogeneous Wireless Networks. International Conference on Communication Technology and System Design. Procedia Engineering, 30, 113-123.

http://dx.doi.org/10.1016/j.proeng.2012.01.841

[6] Kassar, M., Kervella, B. and Pujolle, G. (2008) An Overview of Vertical Handover Decision 
Strategies in Heterogeneous Wireless Networks. Computer Communications, 31, 26072620. http://dx.doi.org/10.1016/j.comcom.2008.01.044

[7] Chuang, M.-C. and Lee, J.-F. (2011) DRO: Domain-Based Route Optimization Scheme for Nested Mobile Networks. EURASIP Journal on Wireless Communications and Networking, 2011, 70. http://dx.doi.org/10.1186/1687-1499-2011-70

[8] Sheikh, K.B., Islam, R., Tahasin, Z.I. and Uddin, R. (2011) An Efficient Handover Scheme for PMIPV6 in IEEE 802.16/WiMAX Network. International Journal of Electrical \& Computer Sciences, 11, 6-14.

[9] Piyakul, T., Thanachai, T., Piboonlit, V. and Pratit, S. (2015) Intelligent Handover Decision Based on Fuzzy Logic for Heterogeneous Wireless Networks. 2015 12th International Conference on Electrical Engineering/Electronics, Computer, Telecommunications and Information Technology, Hua Hin, 24-26 Jun 2015, 1-6.

[10] Farhat, A., Masud, M.H. and Latif, S.A. (2013) Fuzzy Logic Based Handoff Latency Reduction Mechanism in Layer 2 of Heterogeneous Mobile IPv6 Networks. IOP Conference Series. Materials Science and Engineering, 53, Article ID: 012085.

[11] Narayanan, A. and Rajeswari, S. (2014) An Intelligent Vertical Handover Decision Algorithm for Wireless Heterogeneous Networks. American Journal of Applied Sciences, 11, 732-739. http://dx.doi.org/10.3844/ajassp.2014.732.739

[12] Singhrova, A. and Prakash, N. (2011) Vertical Handoff Decision Algorithm for Improved quality of Service in Heterogeneous Wireless Networks. IET Communications, 6, 211-223. http://dx.doi.org/10.1049/iet-com.2010.0820

[13] Fuzzy Logic. www.mathworks.com/access/helpdesk/help/pdf_doc/fuzzy/fuzzy.pdf

[14] Bai, Y. and Wang, D. (2006) Fundamentals of Fuzzy Logic Control-Fuzzy Sets, Fuzzy Rules and Defuzzifications. In: Bai, Y., Zhuang, H. and Wang, D., Eds., Advanced Fuzzy Logic Technologies in Industrial Applications, Springer, Berlin, 17-36. http://dx.doi.org/10.1007/978-1-84628-469-4_2

[15] Sugeno Fuzzy Inference System. http://www.cs.princeton.edu/courses/archive/fall07/cos436/HIDDEN/Knapp/fuzzy004.htm

[16] Tsai, K., Liu, H. and Liu, Y. (2016) Using Fuzzy Logic to Reduce Ping Pong Handover Effect in LTE Networks. Soft Computing, 20, 1683-1694. http://dx.doi.org/10.1007/s00500-015-1655-z

[17] Israt, P., Chakma, N. and Hashem, M.M.A. (2009) A Fuzzy Logic-Based Adaptive Handoff Management Protocol for Next-Generation Wireless Systems. Computer and Information Technology, 4, 288-293. http://dx.doi.org/10.4304/jnw.4.10.931-940

[18] The Network Simulator-NS-2. http://www.isi.edu/nsnam/ns

[19] NIST. NS-2 IEEE802.16 Module. http://www.nist.gov/itl/antd/emntg/ssm_tools.cfm

[20] IEEE Standard 802.21 Framework (2009) IEEE Standard for Local and Metropolitan Area Networks-Part 21: Media Independent Handover.

[21] Huang, K.-L., Chi, K.-H., Wang, J.-T. and Tseng, C.-C. (2013) A Fast Authentication Scheme for WiMAX-WLAN Vertical Handover. Wireless Personal Communications, 71, 555-575. http://dx.doi.org/10.1007/s11277-012-0828-7

[22] Vassiliou, V. and Zinonos, Z. (2009) An Analysis of the Handover Latency Components in Mobile IPv6. Journal of Internet Engineering, 3, 230-240.

[23] Barooah, M., Chakraborty, S., Nandi, S. and Kotwal, D. (2013) An Architectural Framework for Seamless Handoff between IEEE 802.11 and UMTS Networks. Wireless Networks, 19, 411-429. http://dx.doi.org/10.1007/s11276-012-0475-7 
[24] Alkhawlani, M. and Ayesh, A. (2008) Access Network Selection Based on Fuzzy Logic and Genetic Algorithms. Advances in Artificial Intelligence, 2008, Article ID: 793058.

http://dx.doi.org/10.1155/2008/793058

[25] Radhika, K. and Venugopal Reddy, A. (2011) Network Selection in Heterogeneous Wireless Networks Based on Fuzzy Multiple Criteria Decision Making. Electronics Computer Technology, 6, 136-139. http://dx.doi.org/10.1109/icectech.2011.5942067

[26] Attaullah, H. and Iqbal, F. (2008) Intelligent Vertical Handover Decision Model to Improve Qos. 3rd International Conference on Digital Information Management, London, 13-16 November 2008, 119-124. http://dx.doi.org/10.1109/icdim.2008.4746775

[27] Buiati, F., Villalba, L.J.G., Cañas, D.R., Orozco, A.L.S. and Kim, T. (2014) A Zone-Based Media Independent Information Service for IEEE 802.21 Networks. International Journal of Distributed Sensor Networks, 2014, Article ID: 737218.

http://dx.doi.org/10.1155/2014/737218

\section{Submit or recommend next manuscript to SCIRP and we will provide best service for you:}

Accepting pre-submission inquiries through Email, Facebook, LinkedIn, Twitter, etc. A wide selection of journals (inclusive of 9 subjects, more than 200 journals)

Providing 24-hour high-quality service

User-friendly online submission system

Fair and swift peer-review system

Efficient typesetting and proofreading procedure

Display of the result of downloads and visits, as well as the number of cited articles

Maximum dissemination of your research work

Submit your manuscript at: http://papersubmission.scirp.org/ 